\title{
Chorégraphies du corps
}

Cristina 1 inDenmeyer

Le thème du corps, ainsi que la réflexion sur sa sollicitation comme moyen d'expression, n'est pas un thème nouveau au sein de la théorisation psychanalytique.

Dans son rapport, F. Coblence relance le débat en reprenant sa fonction essentielle dans l'élaboration freudienne de l'appareil psychique. Ce qu'elle nous montre avec pertinence et finesse est l'exigence de travail à laquelle le corps participe et soumet la psyché.

Le débat est donc ouvert et la question du dualisme corps'psyché réapparaît. Dans ce débat, impossible de ne pas rappeler la résistance de la part de Freud à la démarche de Groddeck qui tentait de faire du deux, un. Faire du corps et de la psyché un, nous ferait courir le risque de provoquer la disparition de l'essentiel du montage du processus inconscient, « perdant de vue $»^{1}$ l'idée d'un traitement psychique agissant dans un arrière- fond. L'idée de l'arrière- fond représentant toute la dynamique dans laquelle le psychisme se trouve imbriqué dans des situations de manifestation corporelle.

Il sera donc possible de penser que, dans le type de résistance de Freud à la démarche de Groddeck, se maintiendrait la présence du corps dans toute son élaboration théorique. C'est dans ce rappel de la vision freudienne que F. Coblence nous propose son développement théorique. Appuyée sur l'idée d'une unité dualiste, F. Coblence maintient d'un côté la présence du corps, de l'autre la psyché, les deux formant une unité. Toutefois, elle insiste à plusieurs reprises que plus qu'une entité fonctionnant en parallèle avec la psyché, le corps est ce qui permet sa mise en forme. Une forme composée par le corps, la psyché, la sexualité et l'autre : ils sont donc liés par un même mouvement. Cela constitue la position théorique centrale de son rapport.

La rencontre sous des formes diverses : le corps dans la conversion hystérique, le corps érogène, le corps pulsionnel, le corps narcissique ou le moi- corps, témoignent de sa présence nécessaire dans l'évolution de l'élabo. ration de l'appareil psychique. De toutes les déclinaisons qui ont été appréhendées dans les différents rapports de ce congrès, celle que je retiendrai ici,

1. J.- B. Pontalis (1988), «Entre Groddeck et Freud », Perdre de vue, Paris, Gallimard. 
est l'engagement du corps dans les allers et retours entre dedans et dehors, intérieur et extérieur. Ce qui viendrait donner une place au mouvement, psychique, jusqu'à retrouver la participation au corps à corps initial avec l'autre. Par ce mouvement, on retrouve la mise en place de la sexualité et, par voie de conséquence, des processus conflictuels inconscients.

Le corps comme lieu. Le lieu qui en se constituant va intégrer l'intérieur et l'extérieur à la fois. Il n'est pas seulement la limite qui sépare, mais le lieu qui permet la liaison, et également la déliaison de l'assemblage pulsion' nel. C'est sur ce deuxième versant du corps engagé dans la version masochiste que le rapport de S. Savvoloupos mettra l'accent. À ces apports présents dans les rapports, j'ajouterai ceux de J. McDougall et d'A. Green, pour aborder la dimension narcissique présente dans les manifestations corporelles. Les dimensions du dehors et dedans, de l'intérieur et de l'extérieur, de l'absence et de la présence de l'autre sont interrogées par ces deux auteurs.

À la lecture des rapports, nous sommes invités à revoir le statut du corps dans la théorie freudienne. Ce qui suppose que le corps a bien sa place dans cette théorisation, mais également qu'il est parti prenante dans l'échafaudage théorique.

Dans les études sur l'hystérie, nous pouvons souligner une référence plastique au corps. Dans le modèle de la conversion hystérique, c'est l'art de donner forme à l'intérieur qui sera constamment évoqué. Intérieur qui dans l'élaboration freudienne est toujours lié à la représentation des processus inconscients. En défiant et en subvertissant l'anatomie, l'hystérique prend appui sur certaines parties de son corps pour construire une autre scène. C'est cette chorégraphie inconsciente bien orchestrée par des désirs interdits auxquels l'hystérique se livre. La scène présente dans le corps chez l'hystérique viendrait en cacher une autre.

À la question de comment l'hystérique profite de l'occasion donnée par le corps à la mise en scène de ses désirs interdits, Freud répond par l'idée de « complaisance somatique $»^{1}$. F. Coblence revient sur cette notion proposée par Freud et où l'idée du dualisme est soutenue. Quand il y a excitation somati- que, l'occasion se présente pour que viennent se greffer des processus conflic tuels inconscients jusqu'alors inavouables. Les parties du corps d'Élisabeth, Anna, Dora, entre autres, seront donc des occasions données aux interdits conflictuels de trouver un lieu d'expression. L'affect détaché de la représen- tation aboutit à une utilisation de la zone excitée et ainsi à une dynamique fantasmatique qui peut alors s'y installer. nous voulons souligner l'idée de

1. S. Freud (1895 d [1893-1895]), «Études sur l’hystérie », ocf.p, II, Paris, puf, 1967. 
deux aspects hétérogènes qui se retrouvent liés dans un mouvement, permet tant une localisation et la formation du symptôme. Mais ce qui paraît ainsi ébauché c'est la participation de la régression qui donne lieu à des contenus archaïques d'émerger sur la scène : comme s'il fallait un symptôme organique, un moment de rupture, pour que du fantasme puisse advenir.

Des exemples cliniques ne manquent pas pour dire la portée d'une effraction dans le corps qui éveille la nécessité d'un moi sollicité dans ses capacités défensives et créatives. Ferenczi, qui suit l'élaboration freudienne, nous invite dans son texte «Les pathonévroses $»^{1}$ à prendre la mesure de l'importance de cet accordage entre psyché et soma. Dans ses réflexions, la partie du corps douloureuse accueille dans l'excitation de la douleur toute la trame fantasmatique qui saute sur l'occasion pour se livrer à des mouvements inconscients « insoupçonnables ». Dans ce mouvement subversif de l'action du sexuel, le symptôme organique n'est pas seulement passivité, dans sa forme autoérotique, il devient aussi activité. Le corps viendrait être présent au delà de la fonction biologique, par l'action du sexuel dans et avec les manifestations cor porelles qu'il engendre. Dans ce mouvement en action, il y a en premier lieu la place du concept de pulsion pour rendre compte de la dynamique complexe dans laquelle le psychisme et le corps se trouvent imbriqués. Avec le concept de pulsion dans le développement freudien, la place du corps est soulignée comme source en même temps que moyen de décharge et de satisfaction libidinale. Son engagement dans la construction psychique au travers de ses parties utilisées dans les pulsions partielles, et ensuite le corps tout entier, le place dans les scènes fantasmatiques et imaginaires les plus archaïques.

À partir de la seconde topique, l'élaboration freudienne se déplace et « le moi est avant tout un moi corporel $»^{2}$. Par cette élaboration, l'instance du moi se nourrit des liens avec le dedans du corps et le dehors qui le traverse. Le corps n'est pas ici appréhendé dans l'illusion d'un corps fonction, ni dans l'illusion d'un corps image, il est le lieu d'inscription des traces présentes dans les réminiscences, par où le moi va pouvoir se constituer.

Avec la notion de pulsion de mort dans l'évolution de la théorie freudienne, nous arrivons à la fonction inconsciente la plus prometteuse, mais aussi la plus déroutante. Via le masochisme, le corps trouvera une place et une fonction dans la souffrance. Avec le texte "Le problème économique du masochisme $»^{3}$, Freud fait de la maladie l'une des trois solutions qui fonctionnera comme moyen psychique d'arriver à la conclusion d'un arrangement

1. S. Ferenczi (1970), «Les pathonévroses », oc, Pychanalyse II, Paris, Payot, p. 268- 277.

2. S. Freud (1923 b), « Le moi et le ça », ocf.p, xVI, Paris, puf, 1991.

3. S. Freud (1924c), «Le problème économique du masochisme », ocf.p, xVII, Paris, puf, 1992. 
névrotique. Le « besoin de punition » en lien avec " l'incurable culpabilité » ne va pas lâcher facilement. Dans cette proposition de Freud, le décor change, mais le plaisir de la souffrance persiste sous sa forme la plus énigmatique : maintenant elle est déposée dans une partie du corps, dans sa face visible. S. Savvopoulos rappelle cette même idée que «la maladie peut prendre en charge le masochisme $»^{1}$.

Philippe s'adresse à moi alors qu'il a dû subir une fois encore un traitement chimiothérapique pour son cancer des os. Après son premier traitement, tout allait bien, trop bien peut être, jusqu'au jour où à nouveau le médecin lui annonce la présence de cellules cancéreuses. Tout au long des premiè res séances, il me raconte son histoire sous forme d'un discours sans fin, d'une voix monocorde. Il semblait toujours «m'expliquer » qu'il ne pouvait associer quoi que soit sur ce que son cancer pouvait bien évoquer pour lui. Les silences obstinés des séances et mon attente mise à l'épreuve peu à peu lui permettent l'évocation de ce souvenir répétitif : "Quand j'étais petit à la plage, je me suis fait mal au genou. Je saignais beaucoup, donc ma mère m'a amené à un endroit, là bas, où il y avait des pompiers avec des trucs de secours. Il y avait des seringues et des produits. Quand j'ai regardé dehors il y avait un homme, pas un médecin, un homme habillé avec des habits de travail. Il avait une scie. Quand il est passé, il m'a montré la scie et m'a dit : je vais te couper ton genou. »

Dans ses associations, un lien s'établit entre ce souvenir et le fait d'être malade. Cette «complaisance somatique » permet de comprendre comment le corps peut servir d'écran projectif sur lequel se révèle l'image du passé. Pendant les séances suivantes, Philippe va revenir plusieurs fois sur ce souvenir qui apporte des éléments qui seront traités par moi comme des « restes diurnes » dans le rêve. Des « restes diurnes » qui, comme dans le rêve, fonctionnent aussi par une connivence avec la langue : couper le genou est l'acte castrateur redouté que l'homme, pas un médecin, pourrait exécuter sur son corps. Les sensations du corps malade vont servir de support au surgissement de ses théories sexuelles infantiles. Il évoque des relations à un corps libidinalisé puisque l'angoisse de castration déclenchée le fait entrer dans une aire érotique. Ainsi, nous voyons Philippe accéder à un corps différent qui n'est plus le corps médical aplati, spatialisé mais un corps "fait d'histoire », historicisé et reconstruit à partir des restes diurnes où se bâtissent les souvenirs, corps archéologique, pourrait- on dire.

1. S. Savvopoulos (2009), « Les exigences de la représentation », Bulletin de la spp, nº94, p. 182. 
L'érotisation du corps retrouvé par Philippe peut, avec d'autres patients, trouver d'autres voies. La composante autoérotique de la sexualité, nécessaire pour le développement psychique de tout un chacun, peut chez certains patients prendre une forme particulière pour régler leur conflit interne. A. Alexandridis rappelle dans son rapport les procédés autocalmants mis en place par l'enfant pour maîtriser l'excitation. Avec leur corps ou avec des parties de leur corps, le sujet devient autosuffisant pour régler son conflit interne, ce qui peut apparaître, dans la situation analytique, sous des formes diverses.

Souvent ces patients arrivent à l'analyse à condition que leur analyste se dispose à faire un pas en leur direction. Ces démarches, ces exigences contre transférentielles sont apparues dans tous les rapports. M. Aisenstein nous livre, dans sa présentation, ce qui l'amène à des éprouvés d'affects intenses que C. Chabert a nommés « action de l'analyste».

Est- ce que ce recours à cette modalité autoérotique autorisée par son pro pre corps est une façon de lutter contre l'édification d'un cimetière d'objets perdus précocement? Seront ils pris dans des rapports objectaux contaminés par des déceptions et des carences où, sur le mode autoérotique, la satisfaction peut- elle être retrouvée ? N'est- il pas une façon de sauver la relation à toù ce dernier sera protégé de la rage éprouvée, conséquence de la déception?

Cette idée d'un noyau dépressif derrière ses formes défensives est le point de départ de beaucoup des théorisations postérieures : J. McDougall ${ }^{1}$ avec l'idée d'hystérie archaïque, A. Green ${ }^{2}$ avec son travail sur le négatif.

J. McDougall parlera des traces non verbales imprégnées dans le corps. Les manifestations somatiques seront pensées par elle, dans leurs rapports aux premières expériences de l'enfant avec le corps maternel. Le recours à la somatisation sera un mode de défense archaïque, antérieur au langage, et qui résulte de la difficulté de séparation d'avec l'objet. La mère et l'enfant passeront par un processus complexe jusqu'à renoncer à l'unité narcissique originaire dans laquelle ils se trouvent à l'origine. Une des difficultés à laquelle ils seront confrontés, sera de supporter la tension entre le désir de fusion et le désir de séparation. C'est dans cette tension que s'inscrivent le monde intérieur et l'introjection de l'objet pour l'enfant. À partir de sa pratique analytique, J. McDougall propose plusieurs représentations de l'imago maternelle et paternelle qui viennent participer aux mouvements transférentiels de ses patients. Ces représentations peuvent regrouper des angoisses qui font basculer l'enfant dans la psychose ou dans des manifestations somatiques.

1. J. McDougall (1989), Théâtres du corps, Paris, Gallimard.

2. A. Green (1993), Le Travail du négatif, Paris, Éditions de Minuit. 
Dans un autre axe de réflexion, A. Green, grâce à son élaboration sur le travail du négatif, nous parlera d'une forme de destructivité mise au service de l'appareil psychique à des fins défensives. Où on retrouve le paradoxe : l'appareil psychique peut se servir de la destructivité contre la destructivité. L'appareil psychique mis dans des conditions qui lui paraissent insupportables, soit par le comportement de l'objet, soit par ses propres pulsions agressives, peut se construire sur un narcissisme négatif. À l'origine, l'expérience de l'absence de l'objet va permettre à l'enfant la mise en place de sa psyché. Cependant, si des difficultés viennent contaminer ces expériences de présence/ absence alors l'expérience d'absence de l'objet se transforme en menace de disparition de soi, amenant le sujet à trouver une solution qui sera une espèce d'isolement sur un mode autoérotique, une forme de narcissisme en négatif. nous sommes là bien loin du jeu de la bobine... Il s'agit de constructions psychiques mises en place sur l'absence d'investissement libidinal de la part de l'objet, pourtant bien présent. Comme si l'objet s'inscrivait psychiquement sur fond d'une présence en négatif amenant l'enfant à construire une sorte de doublure protectrice de son moi. Cette construction psychique s'organisera sous une forme d'identification en négatif.

Anna était une vomisseuse qui gardait les contenus vomis dans des sacs en plastique et qu'elle disposait dans des coins de sa chambre. Évidemment, sa chambre empestait et c'était dans ces odeurs et ces matières très érotisées qu'Anna se sentait bien. Dans ce mouvement autoérotique en négatif, son corps devient le lieu de mise en scène d'une problématique narcissique d'où seront extraites par elle les questions de son dehors/dedans, son intérieur/extérieur et son rapport avec un père absent et une mère perçue comme trop occupée par elle même pour avoir un regard sur sa fille. Un regard qui ne la voit pas, dit Anna. Ce type de regard Anna le connaît bien. Elle- même en faisait l'expérience quand elle se regardait dans la glace pendant ses cours et entraînements de danse. Des expériences d'hallucinations négatives qui ne pouvaient être stoppées seulement quand Anna, au travers des exercices douloureux que son métier de danseuse lui impose, ressentait la douleur. Comme si le corps pour Anna était sollicité dans une recherche inépuisable d'une garantie de son existence, créant ainsi pour elles ses frontières psychiques. Se penser par son corps, plutôt que penser à l'autre absent, c'est pour Anna faire distraction de sa souffrance. Ses agissements corporels, ses morceaux crachés ou les parties douloureuses de son corps deviennent des lieux de transformation pour ne pas être confronté à l'image du vide. Où nous retrouvons à nouveau le paradoxe signalé dans le rapport de S. Savvopoulos : la souffrance physique qu'Anna s'impose est pour elle synonyme de paix psychique. Cela c'est dire à quel point l'échec de l'identification primaire, de pouvoir se reconnaitre comme 
« moi ». Son corps vomi ou douloureux est investi comme dessinant ses limi- tes et identifié à l'objet décevant et absent, retrouvé dans la douleur et dans ces vomissements, à l'intérieur de soi.

L'interrogation du corps sur plusieurs angles ou «à tous les étages $»^{1}$ selon la formule proposé par F. Coblence : le corps pulsionnel, le corps narcissique, le moi corporel et le corps support du masochisme est la question qu'Anna pose dans cet enchaînement des sollicitations différentes qu'elle vient interro ger. Tout se passait comme si l'émergence des mécanismes archaïques restés refoulés permettait le mouvement régressif présent dans ces sollicitations.

Cristina Lindenmeyer 44, rue de Sévigné 75003 Paris

RÉFÉREnCES BIBLIOGRAPHIQUES

Aisenstein M., Savvopoulos S. (2009), «Les exigences de la représentation », Bulletin de la spp, $\mathrm{n}^{\circ} 94$.

Alexandridis A. (2009), «Enfance : voies, contrats, destinées », Bulletin de la spp, n 94.

Coblence F. (2009), « La vie d'âme. Psyché est corporelle, n'en sait rien », Bulletin de la spp, $\mathrm{n}^{\circ} 94$.

Ferenczi S. (1970), « Les pathonévroses », oc, Pychanalyse II, Paris, Payot.

Freud S. (1895 $d$ [1893- 1895]), «Études sur l'hystérie », ocf.p, II, Paris, puf, 1967.

- (1923 b), «Le moi et le ça », ocf:p, xVI, Paris, puf, 1991.

- (1924c), «Le problème économique du masochisme », ocf.p, xVII, Paris, puf, 1992.

Green A. (1993), Le Travail du négatif, Paris, Éditions de Minuit.

McDougall J. (1989), Théâtres du corps, Paris, Gallimard.

Pontalis J.- B. (1988), «Entre Groddeck et Freud», Perdre de vue, Paris, Gallimard.

1. F. Coblence (2009), « La vie d'âme. Psyché est corporelle, n’en sait rien », Bulletin de la spp, $\mathrm{n}^{\circ} 94$. 